\title{
Force chains and networks: wet suspensions through dry granular eyes
}

\author{
Rangarajan Radhakrishnan ${ }^{1}$ · John R. Royer ${ }^{2}$. Wilson C. K. Poon ${ }^{2} \cdot$ Jin Sun $^{1}$
}

Received: 9 April 2019 / Published online: 27 January 2020

(c) The Author(s) 2020

\begin{abstract}
Recent advances in shear-thickening suspension rheology suggest a relation between (wet) suspension flow below jamming and (dry) granular physics. To probe this connection, we simulated the contact force networks in suspensions of non-Brownian spheres using the discrete element method, varying the particle friction coefficient and volume fraction. We find that force networks in these suspensions show quantitative similarities to those in jammed dry grains. As suspensions approach the jamming point, the extrapolated volume fraction and coordination number at jamming are similar to critical values obtained for isotropically compressed spheres. Similarly, the shape of the distribution of contact forces in flowing suspensions is remarkably similar to that found in granular packings, suggesting potential refinements for analytical mean field models for the rheology of shear thickening suspensions.
\end{abstract}

Keywords Suspension rheology $\cdot$ Granular materials $\cdot$ Network properties $\cdot$ DEM simulations

\section{Introduction}

Suspensions of non-Brownian particles (diameters $d \gtrsim 5 \mu \mathrm{m}$ ) are ubiquitous in industry. Their rheology is critical in the manufacture of numerous formulated products, including paints, ceramics, and cosmetics. Provided the particles are well-stabilised, such suspensions typically shear thicken under flow, where the viscosity $\eta$ increases with increasing stress (or shear rate) $[1,2]$. There is a growing consensus [2-4], based on recent experiments and simulations [5-11], that shear thickening results from the formation of frictional contacts. Friction constrains sliding motion, shifting the jamming volume fraction $\phi_{\mathrm{J}}$ from random close packing, $\phi_{\mathrm{RCP}}$, for frictionless particles at low stress to $\phi_{\mathrm{m}}<\phi_{\mathrm{RCP}}$ for frictional particles at high stress. Full flow curves $\eta(\sigma)$

This article is part of the Topical Collection: In Memoriam of Robert P. Behringer.

John R. Royer

john.royer@ed.ac.uk

1 Institute of Infrastructure and Environment, School of Engineering, The University of Edinburgh, Edinburgh EH9 3JL, UK

2 SUPA and School of Physics and Astronomy, The University of Edinburgh, Edinburgh EH9 3FD, UK can be calculated using the phenomenological Wyart-Cates (WC) model [12], in which the stress-dependent $\phi_{\mathrm{J}}(\sigma)$ interpolates between these two limits, reproducing both simulations and experimental results [13-15].

The importance of static friction and constraints in shear thickening [16] suggests an intimate connection with the physics of dry granular media. Indeed, this connection was evidenced at the macroscopic level with the successful adaptation of constitutive laws for dry flows to describe frictional non-Brownian suspensions [17]. Microscopically, similar features in the spectrum of vibrational modes (density of states) in suspensions and jammed grains have been shown in simplified simulations of frictionless suspensions [18-20], with low frequency 'soft modes' that vanish at a critical coordination number $Z \rightarrow Z_{\mathrm{J}}$, driving the viscosity divergence. This result and the reduction of $Z_{\mathrm{J}}$ in frictional packings $[16,21,22]$ underpins the initial $\mathrm{WC}$ formulation [12], with $\phi_{\mathrm{m}}$ in suspensions corresponding to the limit of random loose packed frictional grains.

Motivated by a lack of obvious structural signatures of jamming in traditional real-space measures, there has been considerable effort devoted to characterizing the statistics and structure of the contact force networks of dry granular materials [23-29]. With limited exceptions [30], contact networks and force distributions in suspensions have received less attention. However, in a 'granular view' of 
shear thickening, force distributions are key [5, 7], particularly at the thickening transition where the form of the force distribution governs the fraction of frictional contacts at a given stress [13, 31].

Here we probe this connection between suspensions and dry grains near jamming in detail, using DEM simulations to model suspensions of spherical particles at varying volume fractions $\phi$ and inter-particle friction coefficients $\mu$. Computing the mean coordination number and force distributions in these simulations, we uncover numerous similarities between flowing suspensions near jamming and dry granular packs, supporting the 'granular view' of suspension rheology.

\section{Methodology}

We simulated 3D suspensions of neutrally-buoyant nonBrownian spheres in a Newtonian background fluid of vis$\operatorname{cosity} \eta_{\mathrm{f}}$. Hydrodynamic forces are calculated using the discrete element method (DEM) [5, 32, 33], including the Stokes drag and short-ranged lubrication interactions. The latter are regularized below a particle separation of $\xi_{\text {min }}$ where $\xi=2 r /\left(d_{1}+d_{2}\right)$ for two particles of diameter $d_{1}, d_{2}$ at a center-to-center distance $r[34,35]$. We neglect longranged hydrodynamic interactions between particles because we only consider high $\phi$.

The regularization of lubrication forces means that particles can come into contact. Such contacts are modeled by a linear Hookean spring $F_{\mathrm{n}}^{c}=k_{\mathrm{n}} h$, with spring constant $k_{\mathrm{n}}$ and the extent of particle overlap $h$. The tangential spring force is $F_{t}^{c}=k_{t} h_{t}$, where $k_{t}=(2 / 7) k_{\mathrm{n}}$ and the incremental tangential stretch $h_{t}$ is initialized to 0 at contact and updated following Silbert et al. [36]. The maximum $F_{t}^{c}$ is set to satisfy the Coulomb criterion, $\left|F_{t}^{c}\right| \leq \mu\left|F_{\mathrm{n}}^{c}\right|$, where $\mu$ is the friction coefficient.

The critical load model (CLM), in which $F_{t}^{c}=0$ when $F_{\mathrm{n}}^{c} \leq F_{\mathrm{CLM}}$, is used to simulate shear thickening [5, 7]. $F_{\mathrm{CLM}}$ mimics the repulsion that prevents facile particle contact $[11,37]$, and sets a force scale for transition between frictionless to frictional flow.

Homogeneous simple shear at rate $\dot{\gamma}$ was imposed by affine deformation with Lees-Edwards periodic boundary condition in LAMMPS [38]. 1872 bi-disperse spheres at diameter ratio 1:1.4 (to prevent crystallization) mixed in equal volumes were simulated [5] at constant $\phi$ and $\dot{\gamma}$ such that the Stokes' number $\rho \dot{\gamma} d^{2} / \eta_{\mathrm{f}}<1$, where $\rho$ is the fluid or particle density.

The total stress was found by summing contributions from the contact, hydrodynamic forces between particles and isolated particle stresslets. The relative viscosity is taken to be $\eta_{\mathrm{s}}=\sigma /\left(\dot{\gamma} \eta_{\mathrm{f}}\right)$, where $\sigma$ is the $x y$ component of the stress tensor. All the results presented were obtained by averaging over at least 10 strain units in steady state. To ensure hardsphere like behavior, in all simulations we verified that the stiffness was set sufficiently high, $k_{\mathrm{n}} \gg \sigma d$, to avoid spurious shear thinning from particle overlaps [39].

\section{Results}

At fixed $\mu$, the suspension's shear rheology is quasi Newtonian. Its $\dot{\gamma}$-independent viscosity increases with $\phi$ and diverges at a $\mu$-dependent jamming volume fraction $\phi_{\mathrm{J}}^{\mu}$, Fig. 1a, which we obtain by fitting

$\eta_{\mathrm{s}}=\alpha^{\mu}\left(\phi_{\mathrm{J}}^{\mu}-\phi\right)^{p}$,

where following literature [15], we fix $p=-2$, leaving $\alpha^{\mu}$ and $\phi_{\mathrm{J}}^{\mu}$ as fitting parameters. As $\mu$ varies from $10^{-4}$ to 10 , $\phi_{\mathrm{J}}^{\mu}$ moved from 0.65 in the low friction limit to 0.57 in the high friction limit, agreeing with previous work $[7,8,15]$. The pre-factor varies weakly with $\mu$ and, despite previous suggestion of $p$ varying somewhat from -2 in the frictionless limit [40], our results are consistent with $p=-2$ at all $\mu$ (Fig. 6). Fits yielded nearly identical $\phi_{\mathrm{J}}^{\mu}$ even if the $p$ is allowed to vary.

As $\phi \rightarrow \phi_{\mathrm{J}}^{\mu}$, the average per-particle contact (or coordination) number $Z$ increases, Fig. 1 b. We estimate $Z$ at jamming, $Z_{\mathrm{J}}^{\mu}$, by linearly extrapolating data for $\phi_{\mathrm{J}}^{\mu}-\phi \leq 0.011$ towards zero. Large fluctuations occurred for $\mu>0.75$ and $\phi_{\mathrm{J}}^{\mu}-\phi \lesssim 0.01$ due to jamming and unjamming transitions in a finite-size system [41]. Thus, we do not extrapolate to find $Z_{\mathrm{J}}^{\mu}$ above $\mu=0.75$.

The plots of $\phi_{\mathrm{J}}^{\mu}$ and $Z_{\mathrm{J}}^{\mu}$ against $\mu$, Fig. 2, are remarkably similar in form to the results from simulations of static, monodisperse sphere packings either under gravity [21] or isotropic compression [22, 42]. Our $\phi_{\mathrm{J}}^{\mu}$ values are slightly higher than those in monodisperse systems, as expected for bi-disperse
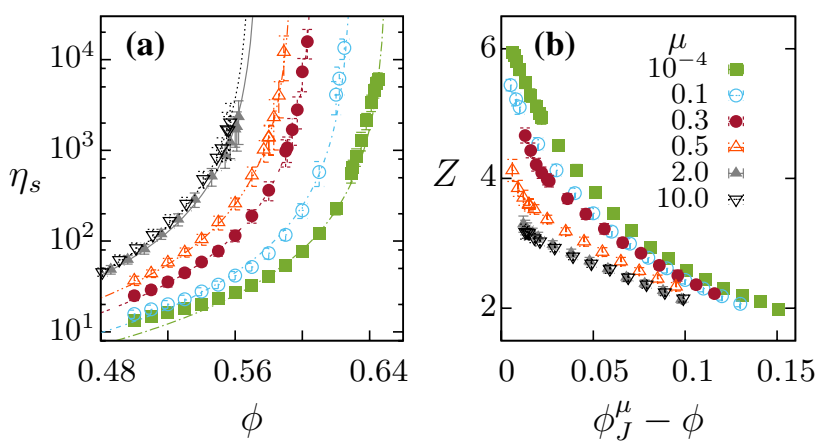

Fig. 1 a Relative viscosity $\eta_{\mathrm{s}}$ of suspensions at different volume fractions $\phi$ and $\mathbf{b}$ the average contact number $Z$ for different particle friction coefficients $\mu$. The jamming packing fraction $\phi_{\mathrm{J}}^{\mu}$ is obtained by fitting $\eta_{s}$ with Eq. 1 indicated by the dot-dashed lines in (a) 


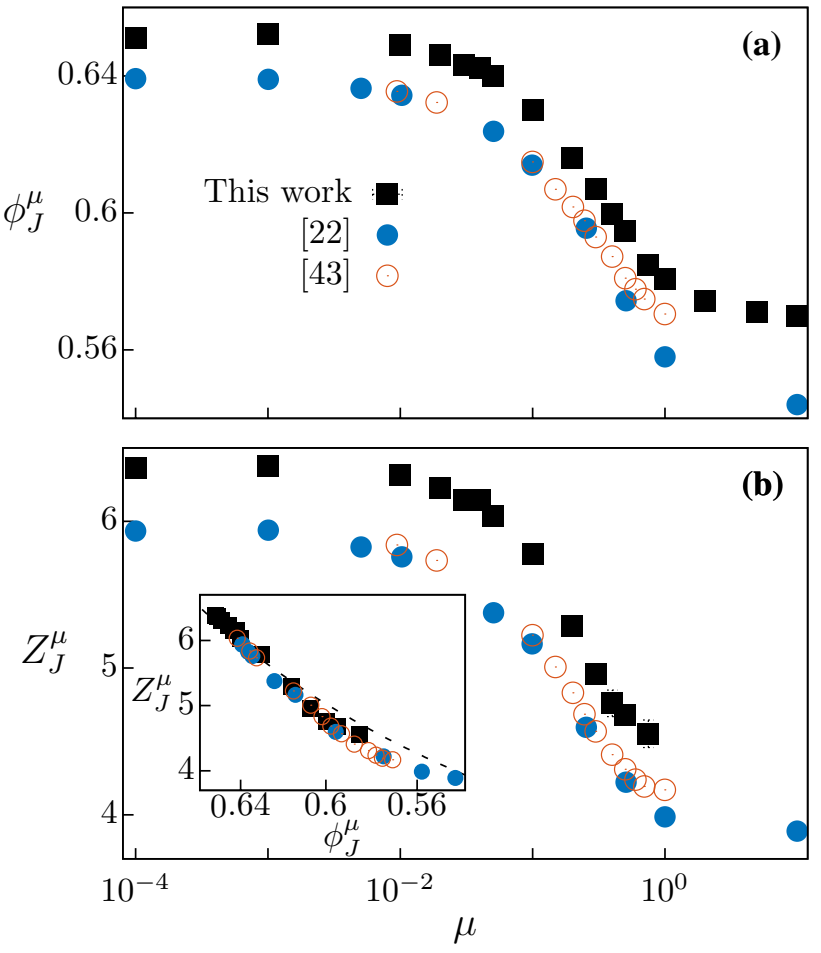

Fig. 2 Dependence of a the critical jamming volume fraction $\phi_{\mathrm{J}}^{\mu}$, and b the critical contact number $Z_{\mathrm{J}}^{\mu}$ of bidisperse suspensions with friction coefficient $\mu$ compared with granular monodisperse spheres [22, 42]. The inset shows the relation between $Z_{\mathrm{J}}^{\mu}$ and $\phi_{\mathrm{J}}^{\mu}$. The dashed line shows Eq. 2 with $q=1.676$

systems [43]. The same is true of $Z_{\mathrm{J}}^{\mu}$, even in the low- $\mu$ limit, where we find $Z_{\mathrm{J}}^{\mu \rightarrow 0} \simeq 6.36$ while the isostatic limit $Z_{\text {iso }}^{\mu=0}=6$ is expected. While increasing particle stiffness slightly reduces $Z_{\mathrm{J}}^{\mu}$, the weak dependence cannot account for the observed excess contact number (Fig. 7). This possibly reflects a difference in how jamming is approached, here from steady-state shear flow under finite stress in contrast to quasi-static dry granular simulations where finite stresses only emerge above jamming. However, we note that other simulation routines also can produce jammed sphere packings slightly above the isostatic limit, even in dry grains [21,44], and emphasize that a more extensive comparison of jamming protocols would be need before attaching physical significance to the slightly increased $Z_{\mathrm{J}}^{\mu}$ values.

For monodisperse spheres, the Edwards statistical ensemble packing theory [45] leads to [46-48]

$\phi_{\mathrm{RLP}}=\frac{Z}{Z+2 q}$

with $q=\sqrt{3} \simeq 1.732$. Eq. 2 fits our data with $Z \rightarrow Z_{\mathrm{J}}^{\mu}$, $\phi \rightarrow \phi_{\mathrm{J}}^{\mu}$ and $q=1.676$, Fig. 2 (inset). We plot the contact number deficit $\Delta Z \equiv Z_{\mathrm{J}}^{\mu}-Z$ against the distance to jamming $\Delta \phi \equiv \phi_{\mathrm{J}}^{\mu}-\phi$ in Fig. 3. In compressed granular packings,

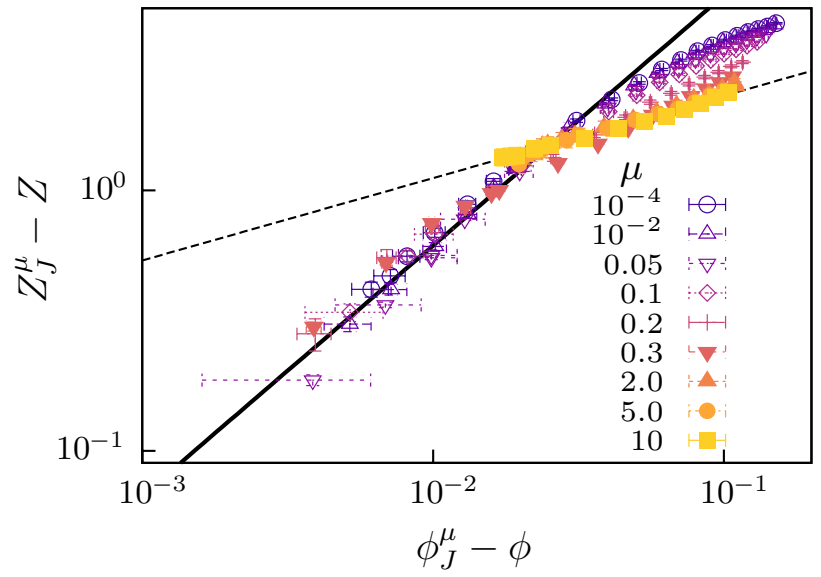

Fig. 3 Contact number deficit $\Delta Z=Z_{\mathrm{J}}^{\mu}-Z$ verses distance to jamming $\Delta \phi=\phi_{\mathrm{J}}^{\mu}-\phi$ for suspensions with varying friction coefficients $\mu$. Lines: fits to $Z_{\mathrm{J}}^{\mu}-Z \sim\left(\phi_{\mathrm{J}}^{\mu}-\phi\right)^{n}$ giving $n=0.96$ (solid) and $n=0.32$ (dashed)

one finds $Z-Z_{\mathrm{J}}^{\mu} \propto\left(\phi-\phi_{\mathrm{J}}^{\mu}\right)^{1 / 2}$ and results for $0 \leq \mu \leq 10$ overlap in the range $10^{-5} \lesssim \Delta \phi \lesssim 10^{-1}$ [22]. The situation in suspensions is more complex, Fig. 3. For $\mu \lesssim 0.06$, our results collapse onto two power law regimes, following $\Delta Z \propto \Delta \phi^{n}$, with $n \approx 1$ below $\Delta \phi \approx 0.06$, and $n \approx 0.3$ beyond.

For $\mu \gtrsim 0.1$, the low $\Delta \phi$ data for different $\mu$ still collapse onto a power-law behavior with $n \approx 1$. Now, however, we no longer find collapse at high $\Delta \phi$. Instead, data at increasing $\mu$ deviate from the $n=1$ power law sooner and drop to a lower asymptote. Data at $\mu \gtrsim 2$ is subject to significant uncertainty because of the impossibility of extrapolating to find $Z_{\mathrm{J}}^{\mu}$ in Fig. 1 b. Instead, we solve for $Z_{\mathrm{J}}^{\mu}$ using Eq. 2 by substituting values of $\phi_{\mathrm{J}}^{\mu}$ obtained previously from fitting Fig. 1a. Including the points so obtained, we find that the data sets for $\mu \gtrsim 0.1$ appear to converge toward a $n=0.3$ power law, the same exponent as the low- $\mu$ data sets, but now with a lower amplitude. It is yet unknown if the power law differs at lower $\Delta \phi$ at the highest $\mu$. These results show that $\Delta Z$ is generally a non-linear function of $\Delta \phi$, and that linearity can only be assumed for suspensions with low particle friction over a very narrow range of volume fractions close to jamming. We note that the non-linearity is robust despite the quantitative uncertainties in $Z_{\mathrm{J}}^{\mu}$ (Fig. 7c).

The rigidity of granular packs is mediated through graingrain contacts, which are distributed heterogeneously in space [49], with some grains bearing many times the mean force while others bear almost none. This is captured by the probability distribution $P(\theta)$, where $\theta \equiv F_{\mathrm{n}} /\left\langle F_{\mathrm{n}}\right\rangle$ is the magnitude of the normal force between neighbors $F_{\mathrm{n}}$ normalized by the mean. In dry granular packing, normal forces arise exclusively from direct contacts. In suspensions, interparticle hydrodynamic interactions also contribute, although 
their exclusion does not change our results (Figs. 11, 12). We calculate $P(\theta)$ from snapshots of the force network collected every 0.1 strain units averaged over 10 strain units, with error bars indicating the standard deviation.

In Fig. 4a we plot $P(\theta)$ for sheared suspensions close to jamming with $\Delta \phi \simeq 5 \times 10^{-3}$ for a range of $\mu$. In common with dry granular packings, we find exponential-like tails at high forces. A closer examination of these high-force tails (Fig. 8) indicates a systematic increase with increasing $\mu$. This increase indicates the formation of stronger contact network for suspensions with high $\mu$, similar to observations from sheared granular spheres [50]. At $\mu=10^{-4}, P(\theta)$ is peaked at $\theta \approx 0.5$. As $\mu$ increases, the probability of lowforce contacts increases, until at $\mu=0.5$ the peak in $P(\theta)$ is no longer evident. An increase in $P(\theta \rightarrow 0)$ with increasing $\mu$ was also reported in simulations of dry granular packings [22]. We observe a similar trend for fixed $\mu=0.2$ at varying $\phi$, with a slight increase in $P(\theta \rightarrow 0)$ as $\Delta \phi$ decreases (Fig. 9). Distributions of the tangential contact forces and the fraction of mobilized contacts are likewise similar to those obtained from dry-grain simulations (Fig. 10).

In compressed dry granular packings, $P(\theta)$ can be described by the empirical relation $[24,26]$

$P(\theta)=a\left(1-b e^{-c \theta^{2}}\right) e^{-\beta \theta}$.

We fit our data to this form, Table 1. The data and fit, Fig. 4a (full line), at $\mu=10^{-4}$ are more pronouncedly peaked than the $P(\theta)$ for compressed amorphous packings of smooth spheres, Fig. 4a (dot-dashed). At $\mu=0.5$, the peak in $P(\theta)$ is no longer evident: $b=0$ and the distribution is purely exponential, Fig. 4a (dashed). Note that our results at high $\mu$ show, and our results at low $\mu$ are consistent with a finite plateau rather than a power-law scaling as $\theta \rightarrow 0[19,23$, $47,51]$.
Table 1 Fit parameters near jamming for $P(\theta)$

\begin{tabular}{lllll}
\hline & $\mathrm{a}$ & $\mathrm{b}$ & $\mathrm{c}$ & $\beta$ \\
\hline$\mu=10^{-4}$ & 1.12 & 0.54 & 33 & 1.0 \\
$\mu \rightarrow \infty$ & 1.12 & 0 & - & 1.0 \\
$\begin{array}{c}\text { Amorphous smooth } \\
\text { spheres [26] }\end{array}$ & 1.5 & 0.59 & 3.1 & 1.21 \\
\hline
\end{tabular}

To examine the force network during shear thickening, we implement a critical load model (CLM), where frictional contacts are activated whenever $F_{\mathrm{n}}$ exceeds a threshold $F_{\mathrm{CLM}}$. For a shear-thickening suspension with $\phi=0.54, \mu=0.5$, our data for $P(\theta)$, Fig. $4 \mathrm{~b}$, are almost identical to those at fixed $\mu$, Fig. $4 \mathrm{a}$, and can be fitted to Eq. 3 in the low- and high-stress limits using the previous low- and high- $\mu$ limit parameters (solid and dashed lines respectively). In the CLM, $F_{\text {CLM }}$ determines the onset stress $\sigma^{*}=F_{\mathrm{CLM}} /\left(1.5 \pi d^{2}\right)$ for shear thickening $[5,7,31]$. The WC jamming volume fraction $\phi_{\mathrm{J}}(\sigma)$ shifts from $\phi_{\mathrm{J}}^{0}$ to $\phi_{\mathrm{J}}^{\mu}$ as the fraction of frictional contacts $f(\sigma)$ increases from $f \rightarrow 0$ for $\sigma \ll \sigma^{*}$ to $f \rightarrow 1$ for $\sigma \gg \sigma^{*}$, giving a thickening flow curve $\eta(\sigma)$.

Typically, $f(\sigma)=\exp \left(-\tilde{\sigma}^{*} / \sigma\right)$ in fitting WC-type models to experiments or simulations $[13,15]$, where $\tilde{\sigma}^{*} \approx \sigma^{*}$ to $\mathcal{O}(1)$. In general, the fraction of frictional contacts is given by

$f=P\left(\theta \geq \theta_{\mathrm{CLM}}\right)=\frac{\int_{\theta_{\mathrm{CLM}}}^{\infty} P(\theta) \mathrm{d} \theta}{\int_{0}^{\infty} P(\theta) \mathrm{d} \theta}$,

where $\theta_{\mathrm{CLM}}(\sigma)=F_{\mathrm{CLM}} /\left\langle F_{\mathrm{n}}(\sigma)\right\rangle$. Assuming $\left\langle F_{\mathrm{n}}\right\rangle \propto \sigma$, we can write $\theta_{\mathrm{CLM}}=\alpha \sigma^{*} / \sigma$, where the pre-factor $\alpha \simeq 1.85$ is found to be independent of $\phi$ (see Fig. 13 and associated discussion). For a simple exponential force distribution,
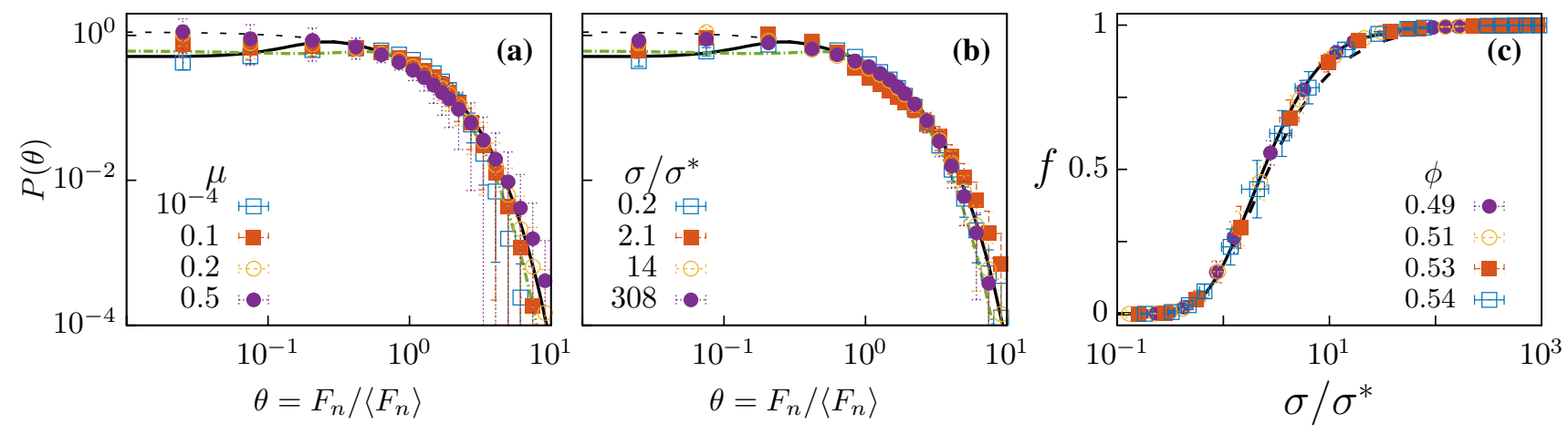

Fig. 4 Probability density functions $P(\theta)$ of the magnitude of the total normal force at contact between particles of suspensions for: a different friction coefficients when $\phi_{\mathrm{J}}^{\mu}-\phi \approx 5 \times 10^{-3}$, b different stresses under the critical load model (CLM) with fixed $\phi=0.54, \mu=0.5$. c The fraction of frictional contacts $f$ with increase in stress for sus- pensions at different $\phi$ with $\mu=0.5$ for the CLM. In (a) and (b), fits to Eq. 3 for $\mu \rightarrow 0$ (solid line), $\mu \rightarrow \infty$ (dashed line), and those from previous experiments [26] (dot-dashed line) with parameters given in Table 1 are shown. The solid and dashed lines in (c) are the $f$ obtained from Eq. 4 for the solid and dashed lines in (a) and (b) with $\alpha=1.85$ 
corresponding to our high- $\mu$ form of Eq. 3 with $b=0$ and $\beta=1$, integrating Eq. 4 gives $f(\sigma)=\exp \left(-\alpha \sigma^{*} / \sigma\right)$, a result previously derived to motivate an exponential form for $f(\sigma)$ $[13,31]$. This procedure can be repeated using the low- $\mu$ fitting parameters in Eq. 3, where $b, c \neq 0$. Interestingly, the resulting $f(\sigma)$ obtained using either low- and high- $\mu$ fitted parameters in Eq. 4, corresponding to the solid and dashed lines in Fig. 4c, differ little; each gives a reasonable account of the data. This reflects the dominant importance of the high-force exponential tail, which remains largely unchanged as either $\mu$ or $\phi$ is varied.

\section{Discussion and conclusions}

We find that sheared dense suspensions exhibit a number of quantitative similarities to dry granular materials near jamming. The $\mu$-dependence of the volume fraction and coordination number at jamming, $\phi_{\mathrm{J}}^{\mu}$ and $Z_{\mathrm{J}}^{\mu}$, are reminiscent of corresponding functions in isotropic sphere packings [22]. Thus, theoretical models relating $\phi_{\mathrm{J}}^{\mu}$ and $Z_{\mathrm{J}}^{\mu}$ in the latter may be extended to suspensions $[46,47]$. The force distribution in suspensions also recalls that in granular packings. An exponential tail dominates in the $\mu \rightarrow 0$ and $\infty$ limits, justifying the use of a simple exponential form for the fraction of frictional contacts in WC-type models of shear thickening.

Despite these similarities, we find that the relation between $\Delta Z$ and $\Delta \phi$ is more complex in suspensions than in dry grains, undercutting a number of assumptions underpinning the WC model for shear thickening. As initially formulated [12], WC drew upon simulations of frictionless hard spheres $[18,19]$, which found $\eta \sim \Delta Z^{-v}$ with $v \sim 2$. The basic physics is that 'soft modes' are lost as the system approaches isostaticity $(\Delta Z \rightarrow 0)$. These soft modes are characterized by the vibrational density of states $D(\omega)$, which gives the number of modes per particle at a given frequency. In dry granular packings above jamming, $D(\omega)$ transitions from classical Debye scaling $\left(\propto \omega^{2}\right.$ in three dimensions) to a constant low-frequency plateau as $\left|Z-Z_{\mathrm{J}}^{\mu}\right| \rightarrow 0$ in both the frictionless and frictional case [16, 52]. A similar change in the shape of $D(\omega)$ was seen in simulated frictionless suspensions as $Z \rightarrow Z_{\mathrm{J}}^{\mu=0}$ [18].

WC assume that the same physics applies in wet frictional suspensions, so that the shape of $D(\omega)$ is likewise controlled by $Z_{\mathrm{J}}^{\mu}$ and the $\eta \sim \Delta Z^{-v}$ scaling applies in both the frictionless and frictional cases. To make useful predictions, they then need to relate the 'natural variable' in dry grains, $Z$, to the 'natural variable' in suspensions, $\phi$, for which they make the further assumption that $\Delta Z \propto \Delta \phi$ in both the low- and high- $\mu$ limits. We find such proportionality only for $\mu \lesssim 0.06$ and very close to jamming ( $\Delta \phi \lesssim 0.03)$, Fig. 2 . In these lowfriction suspensions there is a rollover to a weaker power law at higher $\Delta \phi$, and for higher values of $\mu$ we never reach a regime where $\Delta Z \propto \Delta \phi$.

These discrepancies do not undermine the utility of the WC model as a phenomenological model to analytically capture dense suspension rheology. Indeed, Eq. 1 fits our results over a relatively wide range of $\Delta \phi$ for all values of $\mu$, and the WC model formulated in terms of $\phi$ has proved successful in capturing experimental results $[13,14]$. This suggests that the empirical relation between $\eta$ and $\Delta \phi$ should in fact be viewed as more universal, while the 'soft mode' approach to understanding the viscosity divergence in suspensions may have limited applicability. Indeed, this is in line with work to develop empirical constitutive relations for frictional suspensions by analogy to empirical constitutive relations for flowing grains [17]. It has also recently been shown that viscosity divergence $\eta \propto \Delta \phi^{-2}$ can be derived for suspensions of frictionless spheres through a non-equilibrium kinetic theory approach [53], and this approach could perhaps be extended to frictional suspensions as well.

In our analysis of the force networks in these suspensions, we have neglected the contribution from contact anisotropy to the stress and possible microstructure evolution during shear thickening. However, given the similarities between the force networks in wet suspensions and dry grains, it is likely that more advanced methods used to characterize force networks in jammed packings [54-56] could be applied to open a new window into the rheology and dynamics of dense suspensions.

Acknowledgements The authors acknowledge funding under the UK EPSRC Future Formulations Grant EP/N025318/1. JS was also funded by The Royal Academy of Engineering/The Leverhulme Trust Senior Research Fellowship LTSRF1617/13/2. WCKP was also funded by EPSRC EP/J007404/1. All data used within this publication can be accessed at: https://doi.org/10.7488/ds/2614.

\section{Compliance with ethical standards}

Conflict of interest The authors declare no conflicts of interest associated with this work.

Open Access This article is licensed under a Creative Commons Attribution 4.0 International License, which permits use, sharing, adaptation, distribution and reproduction in any medium or format, as long as you give appropriate credit to the original author(s) and the source, provide a link to the Creative Commons licence, and indicate if changes were made. The images or other third party material in this article are included in the article's Creative Commons licence, unless indicated otherwise in a credit line to the material. If material is not included in the article's Creative Commons licence and your intended use is not permitted by statutory regulation or exceeds the permitted use, you will need to obtain permission directly from the copyright holder. To view a copy of this licence, visit http://creativecommons.org/licenses/by/4.0/. 


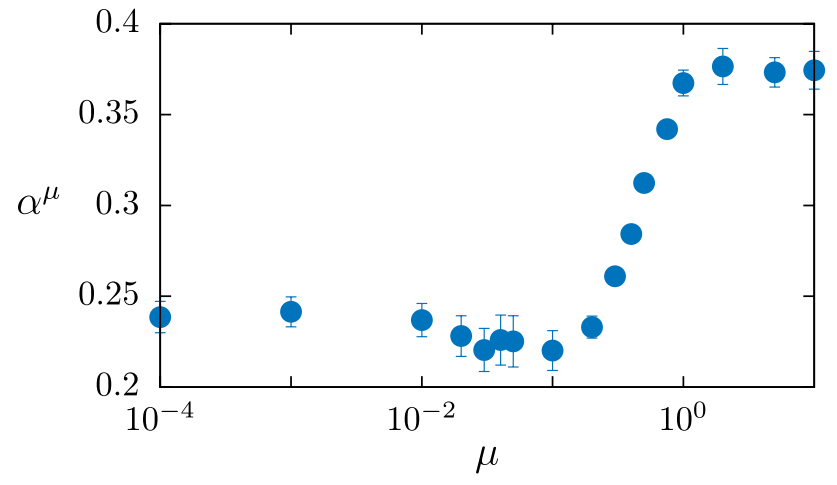

Fig. 5 Dependence of $\alpha^{\mu}$ on $\mu$ obtained from fitting $\eta_{s}$ for different $\phi$ with Eq. 5

\section{Appendix Dependence of $\alpha^{\mu}$ with $\mu$}

The relative viscosities in Fig. 1 of the main text are fit to the expression

$\eta_{s}=\alpha^{\mu}\left(\phi_{\mathrm{J}}^{\mu}-\phi\right)^{p}$,

where following Singh et al. [15], the exponent $p=-2$ is fixed while the pre-factor $\alpha^{\mu}$ is a free fitting parameter. Values of $\alpha^{\mu}$ obtained from these fits vary with $\mu$, transitioning from $\alpha^{\mu \rightarrow 0} \simeq 0.24$ in the low friction limit to $\alpha^{\mu \rightarrow \infty} \simeq 0.37$ in the high friction limit, Fig. 5. These results are quite close to values obtained by Singh et al. [15] in the low friction case, but in the high friction limit they find a slightly higher value $\alpha^{\mu \rightarrow \infty} \simeq 0.5$. The origin of this small difference is unclear, though it could result from subtle differences between the simulation methodologies in this work and in Ref. [15], such as the precise treatment of hydrodynamic interactions.

\section{Relative viscosity scaling near jamming}

To examine the validity of fixing $p=-2$ in Eq. 5, we plot $\eta_{\mathrm{s}} / \alpha^{\mu}$ against $\phi_{\mathrm{J}}^{\mu}-\phi$ in Fig 5. Plotted in this fashion, results for all values of $\mu$ should collapse onto a power law with a slope of -2 . While we find a slightly shallower power law $p \simeq-1.67$ (Fig 5 solid line) away from jamming $\left(\phi_{\mathrm{J}}^{\mu}-\phi \gtrsim 0.1\right)$, close to jamming our results are consistent with $p=-2$ (Fig 5 dotted line). The scaling isn't completely perfect, and for reference we also show $p=-2.25$ (Fig 5 dotted line) as a rough lower bound on $p$. Simulations of frictionless non-Brownian suspensions carried out using Langevin dynamics find a similar change in the power law exponent $p$, from $p=-1.67$ away from jamming to an even lower value $p=-2.55$ close to jamming [40] (Fig. 6).

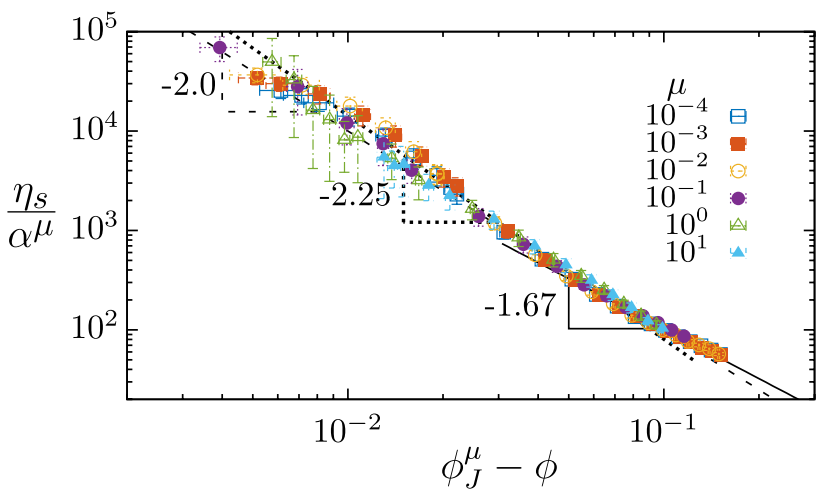

Fig. 6 Power law scaling of the relative viscosity of suspensions near jamming. Solid, dotted and dashed lines show slopes correspond to different power law exponents $p$ in Eq. 5. Note that $p \simeq-2$ as $\phi_{\mathrm{J}}^{\mu}-\phi \rightarrow 0$

\section{Varying particle stiffness}

In soft sphere simulations, such as ours, the jamming volume fractions and contact numbers can depend on the particle spring stiffness $k_{n}$ due to finite particle overlaps [21]. To probe the role of this finite particle compressibility, we vary the dimensionless spring constant $\hat{k}_{n}=\frac{k_{n}}{\eta_{f} \dot{\gamma} d}$, where $d$ is the particle diameter, $\dot{\gamma}$ is the imposed shear rate and $\eta_{f}$ the background fluid viscosity. Results for the viscosity and contact number are shown for various values of $\mu$ are shown for $2 \times$ and $3 \times$ increases in $\hat{k}_{n}$ beyond the nominal value used in the main text $\left(\hat{k}_{n}=3.3 \times 10^{7}\right)$ in Fig. 7. Since stiffer particles require a smaller timestep in DEM simulations, simulations with even stiffer particles become unrealistically burdensome to run.

Figure $7 \mathrm{a}$ shows that the relative viscosity $\eta_{s}$ and the values of $\phi_{J}$ obtained by fitting the results to Eq. 5 do not change significantly with $\hat{k}_{n}$ for the different $\mu$ shown. There is a very weak decrease in the average contact numbers with increasing $\hat{k}_{n}$ for volume fractions close to jamming as shown in Fig. 7b. Thus the extrapolated contact number at jamming $Z_{\mathrm{J}}^{\mu}$ decreases slightly with increasing $\hat{k}_{n}$, but the the inset of Fig. 7c show this trend is only significant for higher friction coefficients, with the split between the three sets of curves above $\phi_{\mathrm{J}}^{\mu} \simeq 0.6$ corresponding to $\mu \gtrsim 0.3$. Thus, as discussed in the main text, the finite particle stiffness cannot account for the offset between $Z_{\mathrm{J}}^{\mu}$ measured in these suspension simulations and $Z_{\mathrm{J}}^{\mu}$ measured by others in dry granular packs. We highlight that this weak stiffness dependance does not impact the relation between $\Delta \phi$ and $\Delta Z$, as shown in Fig. 7c. 

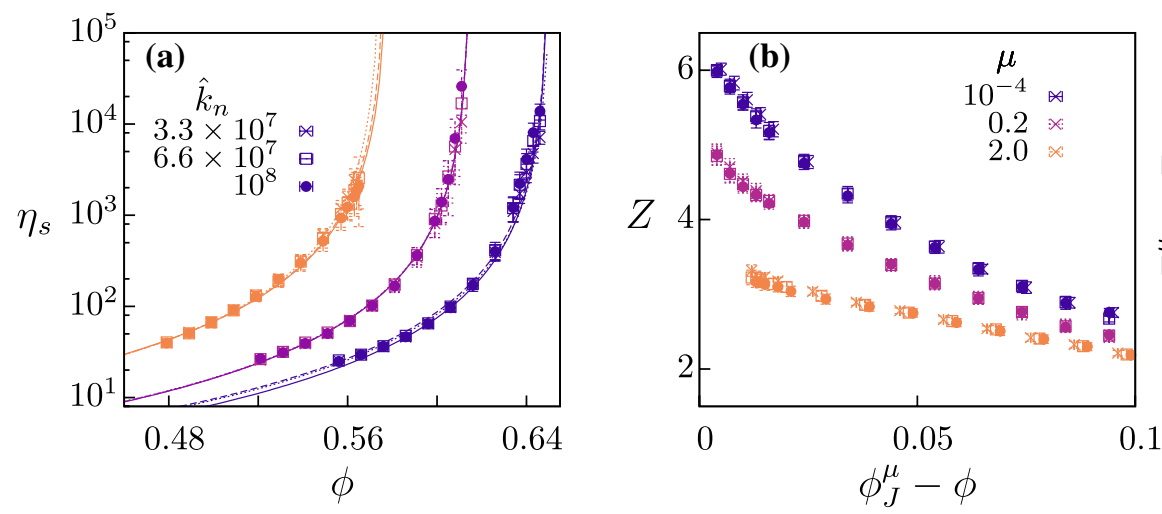

Fig. 7 a Relative viscosity, $\mathbf{b}$ the average contact number and $\mathbf{c}$ the relation of contact number deficit versus distance to jamming of suspensions for 3 different values of spring stiffness $\hat{k}_{n}$ (symbols) and 3 different values of friction coefficients $\mu$ (colors). The inset in (c) shows the extrapolated values of $\phi_{\mathrm{J}}^{\mu}$ and $Z_{\mathrm{J}}^{\mu}$ at different $\mu$ for the

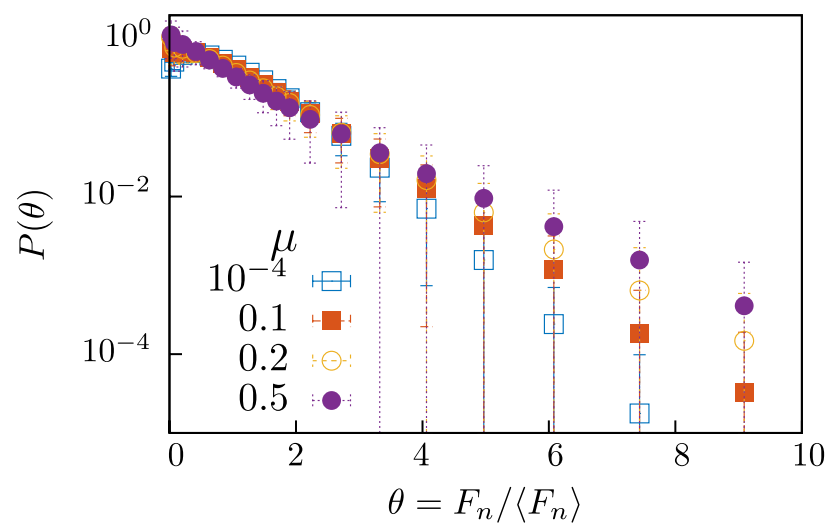

Fig. 8 Probability density function $P(\theta)$ of suspensions for different $\mu$ when $\phi_{\mathrm{J}}^{\mu}-\phi \approx 5 \times 10^{-3}$. Figure $4 \mathrm{a}$ of the manuscript is replotted in a $\log$ linear scale

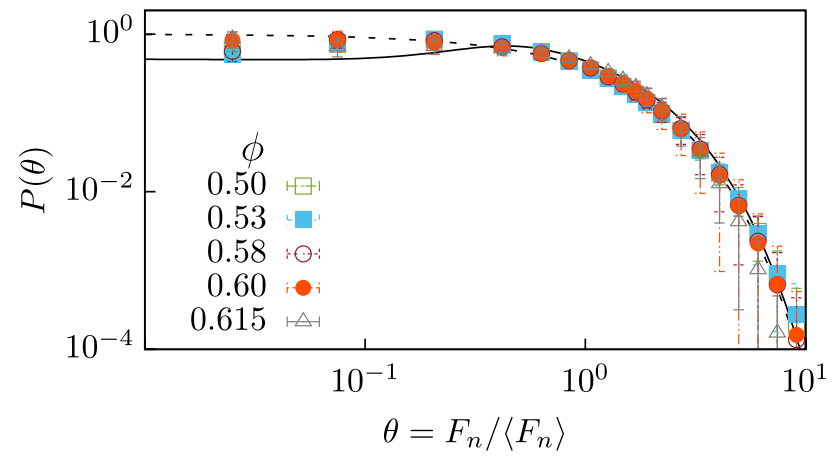

Fig. 9 Probability density function $P(\theta)$ of suspensions for different $\phi$ with a fixed $\mu=0.2$. The solid and the dashed lines are the same as in Fig. $4 a$, b of the manuscript different $\hat{k}_{n}$ shown (symbols). The lines in (a) indicate fits for Eq. 5 for the different $\hat{k}_{n}$. The solid and dashed lines in (c) are the same as Fig. 3 , and the results for $\hat{k}_{n}=3.3 \times 10^{7}$ corresponds to the values shown in Figs. 1, 2 and 3 of the manuscript
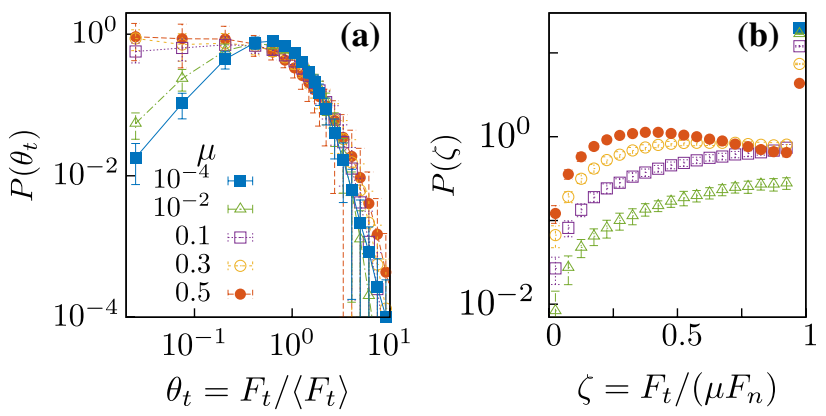

Fig. 10 a Probability density functions of the tangential component of contact force and $\mathbf{b}$ the fraction of mobilized contacts for different $\mu$ when $\phi_{\mathrm{J}}^{\mu}-\phi \approx 5 \times 10^{-3}$

\section{Force distributions}

In Fig. 8 we replot the normal force distributions $P\left(\theta=F_{n} /\left\langle F_{n}\right\rangle\right)$ close to jamming for different $\mu$ (Fig. 4a) on a log-linear scale to highlight the changes to the exponential tail at high forces. For suspensions with volume fractions of close to jamming, we find that there is a systematic increase in the mean probability of large forces with higher $\mu$. Yet, the probability of such high forces are quite low and the values for different $\mu$ are within the error bars of each other.

In Fig. 9 we plot the normal force distributions $P\left(\theta=F_{n} /\left\langle F_{n}\right\rangle\right)$ for different $\phi$ below jamming for fixed $\mu=0.2$. These force distributions are again similar in form to those shown in the main text for both varying $\mu$ at fixed $\phi$ and varying $\sigma$ in a shear thickening suspension with a critical load force (Fig. 4a, b). The solid and dashed lines in Fig. 9 show distributions $P(\theta)$ computed from Eq. (3) in the main text, using the same parameters for $\mu \rightarrow 0$ limit (solid 

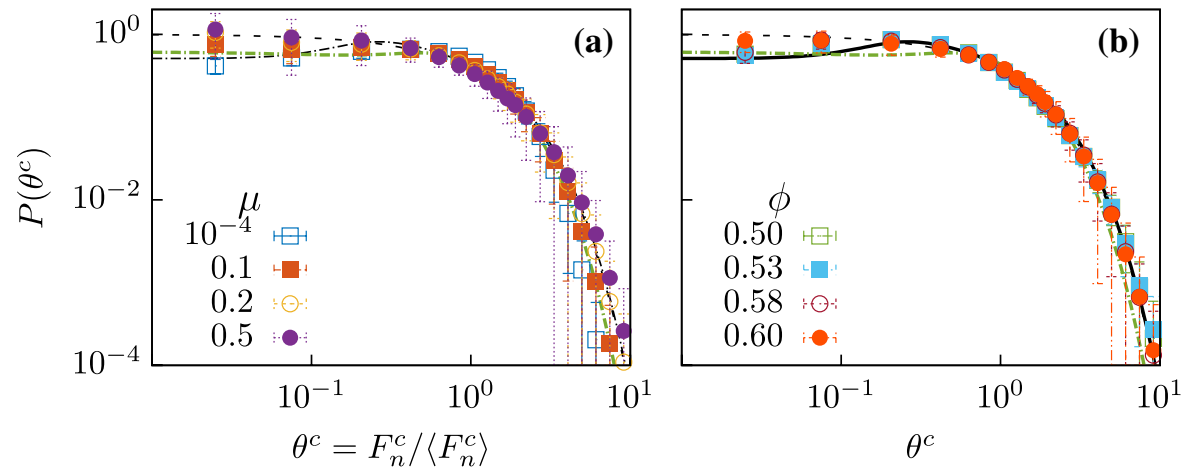

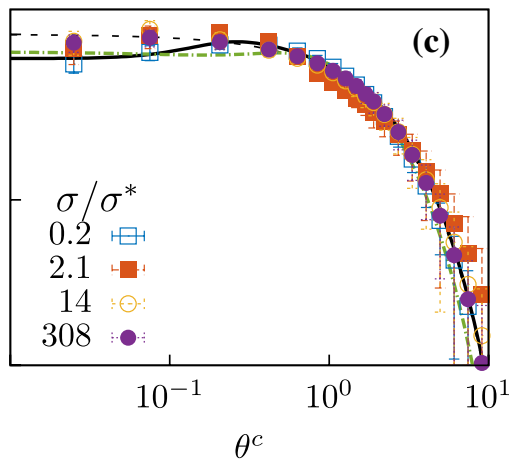

Fig. 11 Probability density functions $P\left(\theta^{c}\right)$ of the contact contribution to the normal force between particles of suspensions for: a different friction coefficients when $\phi_{\mathrm{J}}^{\mu}-\phi \approx 5 \times 10^{-3}$, b different $\phi$ with

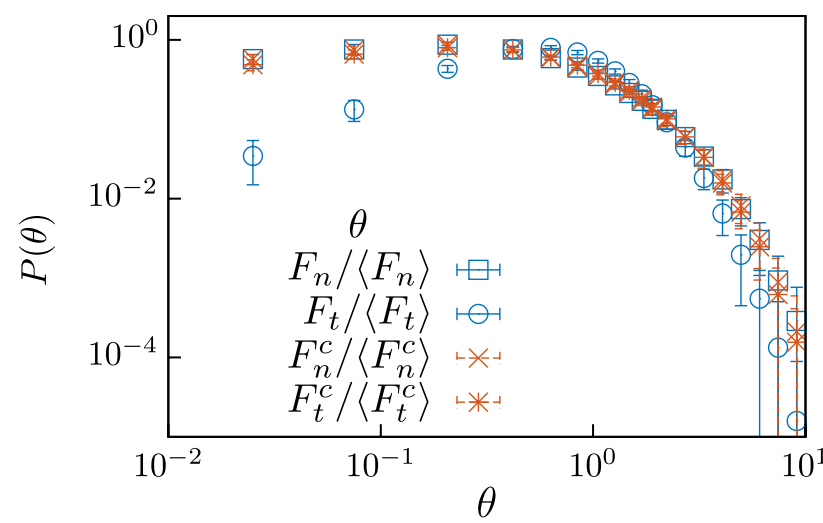

Fig. 12 Comparison of the probability density functions $P(\theta)$ of the pair-wise contact force $\left(F^{c}\right)$ with the total force $(F)$, which includes the hydrodynamic force, for a suspension with $\phi=0.5$, and $\mu=0.2$. Both the normal $\left(F_{n}\right)$ and tangential $\left(F_{t}\right)$ components of the forces are shown

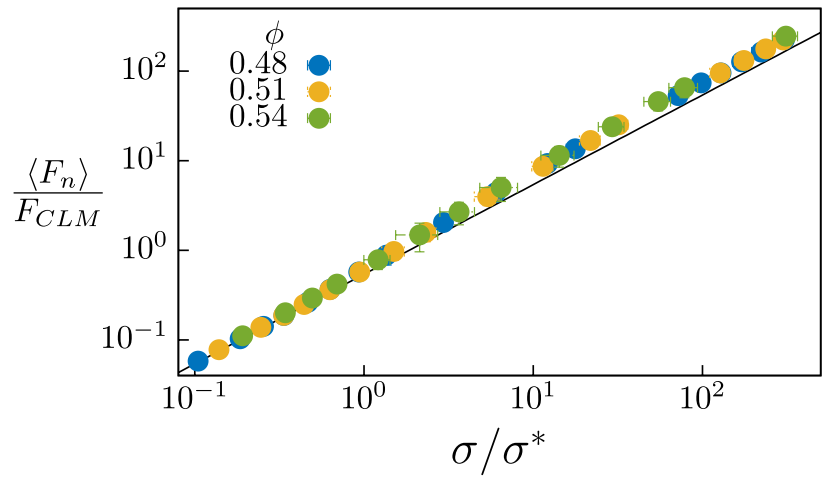

Fig. 13 Relation between $\sigma / \sigma^{*}$ and $\left\langle F_{n}\right\rangle / F_{C L M}$ for different $\phi$ at $\mu=0.5$ for simulations using the critical load model. The solid line corresponds to $\alpha=1.85$

line) and $\mu \rightarrow \infty$ limit (dashed line) given in table 1 in the main text. As $\phi$ increases, there is a slight increase in the a fixed $\mu=0.2$ and $\mathbf{c}$ different stresses under the critical load model with fixed $\phi=0.54, \mu=0.5$. The lines are the same as in Fig. $4 a, b$ of the manuscript

low-force probability, with the peak in $P(\theta)$ transitioning to a plateau.

We also show distributions for the tangential component of the contact forces $F_{t}$ (Fig. 10a), and the plasticity index $\zeta=F_{t} /\left(\mu F_{n}\right)$ (Fig. 10b) from simulations with varying $\mu$ at a fixed distance to jamming $\phi_{\mathrm{J}}^{\mu}-\phi \approx 5 \times 10^{-3}$. In the high force limit, $P\left(\theta_{t}\right)$ exhibits exponential tails at all values of $\mu$, similar to the normal force distributions shown in Fig. 4a from the main text. For low $\mu$, the peak in $P\left(\theta_{t}\right)$ at low forces is more pronounced than in the distribution of normal forces $P(\theta)$, and $P\left(\theta_{t}\right) \rightarrow 0$ as a power law at low $\theta_{t}$ instead of approaching a plateau. For $\mu \gtrsim 0.1, P\left(\theta_{t}\right)$ instead approaches a plateau at low forces, similar to the plateaus observed in $P(\theta)$.

The plasticity distribution $P(\zeta)$ varies significantly with $\mu$. For $\mu=10^{-4}$, nearly all the contacts are at or close to yielding, so that $P(\zeta)$ consists of a sharp peak near $\zeta=1$. As $\mu$ is increased, this peak decreases and the we find progressively more contacts below yielding, with a broad hump developing around $\zeta \simeq 0.25$ for $\mu=0.5$. These results for $P\left(\theta_{t}\right)$ and $P(\zeta)$ in suspensions again mirror results obtained from dry granular packings [21, 22, 57], highlighting further similarities between the force networks in these different types of systems.

\section{Contact force distributions}

The force distributions in the main text, Fig. $4 a$, b, were computed including both hydrodynamic and contact contributions to the pairwise forces between neighboring particles. We can also restrict ourselves to the contributions from grain-grain contacts $F^{c}$, arising from the small but finite overlaps between particles. We show distributions of the direct contact forces $P\left(\theta^{c}\right)$, with $\theta^{c}=F_{\mathrm{n}}^{c} /\left\langle F_{\mathrm{n}}^{c}\right\rangle$, for varying $\mu$ (Fig. 11a), varying $\phi$ (Fig. 11b) and varying stress in CLM simulations (Fig. 11c), analogous to the distributions 
$P(\theta)$ shown here and in the main text. To compare against the probability distributions for the total pairwise force, we also plot $P(\theta)$ computed from Eq. 3 in the main text using same three sets of parameters from Table 1 . We find that these contact-only force distributions are nearly identical, reflecting the dominant role played by contact forces in dense suspensions.

The hydrodynamic force contributions of particles near contact can be negative and can decrease the probability at low forces. A comparison of the probability distributions of the contact force to the total force reveals that only the probability of the tangential forces are affected by the small negative hydrodynamic forces (Fig. 12). The impact of hydrodynamic interactions on the normal forces are perhaps relegated to very small forces and do not affect the results shown in the manuscript

\section{Relating bulk stress and contact mean force}

In the main text, to connect the measured force distributions $P(\theta)$ to the fraction of frictional contacts $f(\sigma)$ in shear thickening suspensions, we assume that the mean normal force is proportional to the bulk suspension stress, allowing us to write $\theta_{\mathrm{CLM}}=F_{\mathrm{CLM}} /\langle F\rangle=\alpha \sigma^{*} / \sigma$. This is a simplifying assumption, in general we should expect $\alpha$ to vary as both the suspension microstructure and the precise shape of $P(\theta)$ evolve through the shear thickening transition. However, we find this linear relation roughly holds, Fig. 13 , with $\alpha \simeq 1.85$ in the low stress limit, independent of $\phi$. Using a slightly lower value of $\alpha$, consistent with fitting the data in Fig. 13 in either the intermediate- or high-stress limit, has a limited impact when computing $f(\sigma)$ from an analytic expression for $P(\theta)$ as in Fig. 4 of the main text.

\section{References}

1. Barnes, H.A.: Shearthickening ("dilatancy") in suspensions of nonaggregating solid particles dispersed in newtonian liquids. J. Rheol. 33(2), 329 (1989)

2. Denn, M.M., Morris, J.F.: Soft matter shear thickening in concentrated suspensions of smooth spheres in Newtonian suspending fluids. Soft Matter 14, 170 (2017)

3. Morris, J.F.: Lubricated-to-frictional shear thickening scenario in dense suspensions. Phys. Rev. Fluids 3, 110508 (2018)

4. Guazzelli, É., Pouliquen, O.: Rheology of dense granular suspensions. J. Fluid Mech. 852, P1 (2018)

5. Seto, R., Mari, R., Morris, J.F., Denn, M.M.: Discontinuous shear thickening of frictional hard-sphere suspensions. Phys. Rev. Lett. 111(21), 218301 (2013)

6. Fernandez, N., Mani, R., Rinaldi, D., Kadau, D., Mosquet, M., Lombois-Burger, H., Cayer-Barrioz, J., Herrmann, H.J., Spencer, N.D., Isa, L.: Microscopic mechanism for shear thickening of non-Brownian suspensions. Phys. Rev. Lett. 111, 108301 (2013)

7. Mari, R., Seto, R., Morris, J.F., Denn, M.M.: Shear thickening, frictionless and frictional rheologies in non-Brownian suspensions. J. Rheol. 58(6), 1693 (2014)

8. Gallier, S., Lemaire, E., Peters, F., Lobry, L.: Rheology of sheared suspensions of rough frictional particles. J. Fluid Mech. 757, 514 (2014)

9. Lin, N.Y.C., Guy, B.M., Hermes, M., Ness, C., Sun, J., Poon, W.C.K., Cohen, I.: Hydrodynamic and contact contributions to continuous shear thickening in colloidal suspensions. Phys. Rev. Lett. 115, 228304 (2015)

10. Clavaud, C., Bérut, A., Metzger, B., Forterre, Y.: Revealing the frictional transition in shear-thickening suspensions. Proc. Natl. Acad. Sci. USA 114(20), 5147 (2017)

11. Comtet, J., Chatte, G., Nigues, A., Bocquet, L., Siria, A., Colin, A.: Pairwise frictional profile between particles determines discontinuous shear thickening transition in non-colloidal suspensions. Nat. Commun. 8, 15633 (2017)

12. Wyart, M., Cates, M.E.: Discontinuous shear thickening without inertia in dense non-Brownian suspensions. Phys. Rev. Lett. 112(9), 098302 (2014)

13. Guy, B.M., Hermes, M., Poon, W.C.K.: Towards a unified description of the rheology of hard-particle suspensions. Phys. Rev. Lett. 115, 088304 (2015)

14. Royer, J.R., Blair, D.L., Hudson, S.D.: Rheological signature of frictional interactions in shear thickening suspensions. Phys. Rev. Lett. 116(18), 188301 (2016)

15. Singh, A., Mari, R., Denn, M.M., Morris, J.F.: A constitutive model for simple shear of dense frictional suspensions. J. Rheol. 62(2), 457 (2018)

16. van Hecke, M.: Jamming of soft particles: geometry, mechanics, scaling and isostaticity. J. Phys. Condens. Matter 22(3), 033101 (2010)

17. Boyer, F., Guazzelli, É., Pouliquen, O.: Unifying suspension and granular rheology. Phys. Rev. Lett. 107(18), 188301 (2011)

18. Lerner, E., Düring, G., Wyart, M.: A unified framework for nonBrownian suspension flows and soft amorphous solids. Proc. Natl. Acad. Sci. USA 109(13), 4798 (2012)

19. Lerner, E., Düring, G., Wyart, M.: Toward a microscopic description of flow near the jamming threshold. Europhys. Lett. 99(5), 58003 (2012)

20. Düring, G., Lerner, E., Wyart, M.: Length scales and self-organization in dense suspension flows. Phys. Rev. E 89, 022305 (2014)

21. Silbert, L.E., Ertaş, D., Grest, G.S., Halsey, T.C., Levine, D.: Geometry of frictionless and frictional sphere packings. Phys. Rev. E 65, 031304 (2002)

22. Silbert, L.E.: Jamming of frictional spheres and random loose packing. Soft Matter 6, 2918 (2010)

23. Radjai, F., Jean, M., Moreau, J.J., Roux, S.: Force distributions in dense two-dimensional granular systems. Phys. Rev. Lett. 77, 274 (1996)

24. Mueth, D.M., Jaeger, H.M., Nagel, S.R.: Force distribution in a granular medium. Phys. Rev. E 57, 3164 (1998)

25. Makse, H.A., Johnson, D.L., Schwartz, L.M.: Packing of compressible granular materials. Phys. Rev. Lett. 84, 4160 (2000)

26. Blair, D.L., Mueggenburg, N.W., Marshall, A.H., Jaeger, H.M., Nagel, S.R.: Force distributions in three-dimensional granular assemblies: effects of packing order and interparticle friction. Phys. Rev. E 63, 041304 (2001)

27. Snoeijer, J.H., Vlugt, T.J.H., van Hecke, M., van Saarloos, W.: Force network ensemble: a new approach to static granular matter. Phys. Rev. Lett. 92, 054302 (2004)

28. Majmudar, T.S., Behringer, R.P.: Contact force measurements and stress-induced anisotropy in granular materials. Nature 435, 1079 (2005) 
29. Corwin, E.I., Jaeger, H.M., Nagel, S.R.: Structural signature of jamming in granular media. Nature 435, 1075 (2005)

30. Melrose, J.R., Ball, R.C.: "Contact networks" in continuously shear thickening colloids. J. Rheol. 48(5), 961 (2004)

31. Ness, C.J., Sun, J.: Shear thickening regimes of dense non-Brownian suspensions. Soft Matter 12, 914 (2016)

32. Ball, R., Melrose, J.: A simulation technique for many spheres in quasi-static motion under frame-invariant pair drag and Brownian forces. Physica A 247(1), 444 (1997)

33. Ness, C.J., Sun, J.: Flow regime transitions in dense non-Brownian suspensions: rheology, microstructural characterization, and constitutive modeling. Phys. Rev. E 91, 012201 (2015)

34. Cheal, O., Ness, C.: Rheology of dense granular suspensions under extensional flow. J. Rheol. 62(2), 501 (2018)

35. Radhakrishnan, R.: Simplification of lubrication forces for unequal spheres. Technical report (2018). https://doi.org/10.5281/ zenodo. 1137305

36. Silbert, L.E., Ertaş, D., Grest, G.S., Halsey, T.C., Levine, D., Plimpton, S.J.: Granular flow down an inclined plane: Bagnold scaling and rheology. Phys. Rev. E 64, 051302 (2001)

37. Chatte, G., Comtet, J., Nigues, A., Bocquet, L., Siria, A., Ducouret, G., Lequeux, F., Lenoir, N., Ovarlez, G., Colin, A.: Shear thinning in non-Brownian suspensions. Soft Matter 14, 879 (2018)

38. Plimpton, S.: Fast parallel algorithms for short-range molecular dynamics. J. Comput. Phys. 117(1), 1 (1995)

39. Radhakrishnan, R., Sun, J.: Shear thinning beyond shear thickening from discrete element method simulations of dense nonBrownian suspensions (2019) (under preparation)

40. Kawasaki, T., Coslovich, D., Ikeda, A., Berthier, L.: Diverging viscosity and soft granular rheology in non-Brownian suspensions. Phys. Rev. E 91, 012203 (2015)

41. Banigan, E.J., Illich, M.K., Stace-Naughton, D.J., Egolf, D.A.: The chaotic dynamics of jamming. Nat. Phys. 9, 288-292 (2013)

42. Sun, J., Sundaresan, S.: A constitutive model with microstructure evolution for flow of rate-independent granular materials. J. Fluid Mech. 682, 590 (2011)

43. Pednekar, S., Chun, J., Morris, J.F.: Bidisperse and polydisperse suspension rheology at large solid fraction. J. Rheol. 62(2), 513 (2018)

44. Koeze, D.J., Vågberg, D., Tjoa, B.B.T., Tighe, B.P.: Mapping the jamming transition of bidisperse mixtures. Europhys. Lett. 113(5), 54001 (2016)
45. Edwards, S., Oakeshott, R.: Theory of powders. Physica A 157(3), 1080 (1989)

46. Song, C., Wang, P., Makse, H.A.: A phase diagram for jammed matter. Nature 453, 629 (2008)

47. Wang, P., Song, C., Briscoe, C., Wang, K., Makse, H.A.: From force distribution to average coordination number in frictional granular matter. Physica A 389(19), 3972 (2010)

48. Baule, A., Morone, F., Herrmann, H.J., Makse, H.A.: Edwards statistical mechanics for jammed granular matter. Rev. Mod. Phys. 90, 015006 (2018)

49. Jaeger, H.M., Nagel, S.R., Behringer, R.P.: Granular solids, liquids, and gases. Rev. Mod. Phys. 68(4), 1259 (1996)

50. Singh, A., Magnanimo, V., Luding, S.: In: Hicks, M., Brinkgreve, R., Rohe, A. (eds.) NUMGE 2014, pp. 409-419. CRC Press, Boca Raton (2014). ISBN 978-1-138-00156-6

51. Kyeyune-Nyombi, E., Morone, F., Liu, W., Li, S., Gilchrist, M.L., Makse, H.A.: High-resolution of particle contacts via fluorophore exclusion in deep-imaging of jammed colloidal packings. Physica A 490, 1387 (2018)

52. Henkes, S., van Hecke, M., van Saarloos, W.: Critical jamming of frictional grains in the generalized isostaticity picture. Europhys. Lett. 90(1), 14003 (2010)

53. Suzuki, K., Hayakawa, H.: Theory for the rheology of dense nonBrownian suspensions: divergence of viscosities and $\mu$-j rheology. J. Fluid Mech. 864, 1125 (2019)

54. Sarkar, S., Bi, D., Zhang, J., Behringer, R.P., Chakraborty, B.: Origin of rigidity in dry granular solids. Phys. Rev. Lett. 111, 068301 (2013)

55. Thomas, J.E., Ramola, K., Singh, A., Mari, R., Morris, J.F., Chakraborty, B.: Microscopic origin of frictional rheology in dense suspensions: correlations in force space. Phys. Rev. Lett. 121, 128002 (2018)

56. Kollmer, J.E., Daniels, K.E.: Betweenness centrality as predictor for forces in granular packings. Soft Matter 15, 1793 (2019)

57. Landry, J.W., Grest, G.S., Silbert, L.E., Plimpton, S.J.: Jamming of frictional spheres and random loose packing. Phys. Rev. E 67, 041303 (2003)

Publisher's Note Springer Nature remains neutral with regard to jurisdictional claims in published maps and institutional affiliations. 